\title{
Verifying Statemate Statecharts Using CSP and FDR
}

\author{
A.W. Roscoe and Z. $\mathrm{Wu}^{\star}$ \\ Oxford University Computing Laboratory \\ \{bill.roscoe, zhenzhong.wu\}@comlab.ox.ac.uk
}

\begin{abstract}
We propose a framework for the verification of statecharts We use the CSP/FDR framework to model complex systems designed in statecharts, and check for system consistency or verify special properties within the specification. We have developed an automated translation from statecharts into CSP and exploited it in both theoretical and practical senses.
\end{abstract}

\section{Introduction}

Statecharts are a popular means for designing the hierarchical state machines which are used in embedded systems, telecommunications etc. Clarke and his colleagues have developed the SMV tool for checking finite state systems against specifications in the temporal logic CTL [4-6]. Work by Bienmuller, Damm and their colleagues has built up the STVE to model and verify some industrial applications $[2,3,7,11]$.

The CSP/FDR framework is well established as a methodology for analyzing interacting systems and state machines [25]. A number of people have done prototyping work in the translation of statechart problems in a form the FDR can analyze. Work by Fuhrmann and his colleagues [14] shows how a subset of the statechart is expressed and verified by CSP/FDR. In this paper we report on our development of an automated translation from Harel's Statemate Statecharts into CSP, and exploit it in both theoretical and practical senses, mainly in defining language semantics and in system verification.

The statecharts formalism derives from conventional finite state machines. It is a structured analysis approach for modelling reactive systems. The Statemate semantics of statecharts was introduced by David Harel [18], and has been proved to be very useful for specifying concurrent systems. It supports both models of timing: synchronous and asynchronous. Verification techniques for statecharts have often been based on extensive checking or simulation. However, the informality of such approaches can easily lead to important requirements being overlooked and, since testing is rarely exhaustive, failures can be missed.

Our approach to Statemate Statecharts uses the CSP process algebra to specify concurrent systems. A statechart can be represented as a CSP process;

\footnotetext{
* Supported by QinetiQ.
} 
statechart constructs such as hierarchy, AND and OR states, and communications all having CSP analogues. CSP-based tools such as FDR can then be used to verify properties of statecharts by performing refinement checks on the translation.

We initially adopted our approach as a way of understanding the semantics of statecharts, but it proved unexpectedly successful at verifying practical systems. We have therefore sought to include as wide a range of statechart constructs as possible in our compiler. In this paper we document which constructs are covered, but only give technical details of the more important ones. The paper is concluded by a case study.

\section{Modelling Statecharts in CSP}

Before we can describe the simulation we need to understand the basic concept of Statemate Statecharts.

\subsection{Statemate Semantics}

The statechart concept is based fundamentally on three ideas:

- Hierarchy: a statechart can exist within a single state of a higher level state machine.

- State machines: the basic component of a statechart is a sequential state machine, with guarded actions between states that have the potential to set signals and assign to shared variables.

- Parallelism (AND states): having several sequential machines running side by side.

There are many semantics of statecharts. One of the most important is the Statemate Statecharts of Harel [18], as refined by various developers. The various semantics of statecharts take different views on, for example:

- Concurrency. Statemate has an eager and concurrent model of AND states - if several states can proceed on one step then they all do.

- Timing model. Statemate has a two-level timing model and expects a system to settle through a number of "small" time steps before allowing further external signals to be processed.

- Nondeterminism. Statemate expects (under priority) at most one action of each OR-state to be available at one time, and forbids race conditions on variables.

- Priority. Statemate gives the highest priority to actions that are enabled further up the hierarchy, together with various other rules.

The statechart diagram in Fig. 1 illustrates the uses of these features in Statemate semantics. This statechart module has two input events: event1 and event2. There are two variables: $v 1$ and v2. There is one constant Limit. Suppose, 


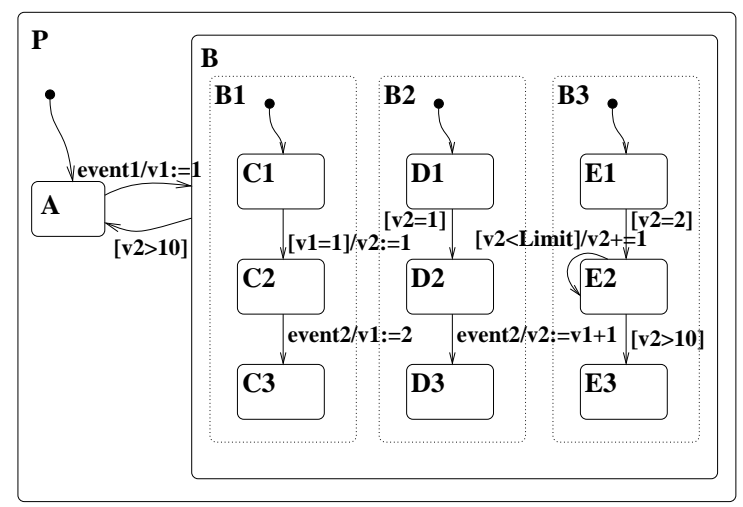

Fig. 1. Example Statemate Statechart

for example, that state $A$ is active, Limit is set to 11 , and two variables are set to 0 . If event 1 occurs under these conditions, the transition from state $A$ to state $B$ is enabled, and variable $v 1$ is set to 1 .

State $B$ is an AND-state: states $B 1, B 2$ and $B 3$ are its sub-states. Once an AND-state is entered, all its components become active in parallel. In Fig. 1, states $B 1, B 2, B 3$ are entered simultaneously when entering $B$ so that all three sub-states become active. Once they are entered transitions emerge from Default Connectors automatically. States $C 1, D 1$ and E1 are activated.

Under the asynchronous time model, time is not advanced at every single step but at super-steps. Each super-step consists of a collection of steps. Execution of a series of steps within one super-step does not advance the timer, these steps take place at the same point of time without introducing any external changes from the environment. Once the system is in a stable state, i.e., when no enabled transitions exist in the system, a super-step is completed. The environment can generate signals to enable new transitions and subsequently execute another super-step. In Fig. 1, the transition from $C 1$ to $C 2$ is taken, setting v2 to 1. The transition from $D 1$ to D2 is enabled and taken afterwards. There are no more enabled transitions at this point and the system becomes stable. So this super-step is completed, then the environment generates event2 to trigger new transitions.

When multiple actions make changes on one element in the same step, we cannot predict the outcome and this is considered an error. There is a potential danger from reading and writing the same element in different parallel threads; however Statemate semantics are that an assignment to an element does not take effect till next step. In Fig. 1, occurrence of event2 enables both transitions from $C 2$ to $C 3$ and from $D 2$ to $D 3$. There are two actions available at the same time: $v 1$ will attain the value of 2 and $v 2$ will be assigned to " $v 1+1$ ". Without the delay described above this would lead to ambiguity. The signals generated and data-items changed cannot take effect until the completion of the step. This 
suggests that $v 1$ still and always holds the value 1 in this step so that $v 2$ will become 2 but not 3 after the assignment " $v 2$ : $=v 1+1$ ".

There is a conflict if multiple transitions are enabled from one common state. Those transitions cannot be performed in the same step. Nondeterminism occurs when there are some conflicts and those transitions within each conflict have the same priority. The choice of transitions results in different statuses. Even if two enabled transitions lead to the same state, non-determinism still occurs due to the changes of some other items during the transition, for instance, different signals being generated. In this case the overall result states will be different. In Fig. 1, transition from state $E 1$ to state $E 2$ is enabled since the condition is fulfilled, leading to the activation of state E2. The self-loop transition is enabled until v2 reaches 11 . There is a potential conflict at this point, three transitions enabled at the same time: the transition pointed to E2 itself, the transition from state E2 to state E3, and the transition from state $B$ to state $A$.

A transition from a lower level state to a higher-level state takes priority over other types of transitions from the same state. This phenomenon, also called "Preemptive Interrupt" [18], happens when a high level transition prevents transitions on lower levels. Transition priority provides a way to pre-determine one transition among a group of enabled transitions and also to avoid the potential nondeterminism. In Fig. 1, the transition from state $B$ to state $A$ has the highest priority among all three, and so is preferred. There will be no non-determinism in this case. If none of conflicting multiple transitions has higher priority than the others, different semantics treat it in different ways. Some semantics introduce a specific priority of execution. For example, in Matlab Stateflow, transitions are taken according to their relative locations in the statechart diagram in clockwise order starting at the "twelve o'clock" position. ${ }^{1}$ Our compiler treats nondeterminism as an error which would be returned by the system. This could be altered straightforwardly, but in some cases it would lead to our tool being less efficient as it presently exploits the determinism of statecharts. ${ }^{2}$

\subsection{Compiler}

The compiler is written as a program in $\mathrm{CSP}_{M}$ [25] making heavy use of functional programming. It consists of three main parts, plus some other functions:

- The mechanism to construct a single sequential chart: each individual chart is described as a transition system and a set of state labels indicate its substates.

- The mechanism to construct a hierarchy of individual sequential statecharts with capability of promotion: the overall hierarchical structure of the system is expressed as a special tree data type; all charts are built based on the root. Charts with no subcharts are expressed as single-leaf trees.

\footnotetext{
${ }^{1}$ See [Mathworks97] for a precise description of the evaluation order [22].

2 The full description of the syntax of Statemate Statecharts is beyond the scope of this paper. I refer the interested readers to a full description in Harel and Naamad [18].
} 
- Constructing variables and timers as processes running in parallel with the main system.

\subsection{Timing Model and Step Semantics}

Two levels of timing model are supported in the compiler. A small step is indicated by event step, and event tock represents a super-step. The entire system created has a synchronous timing model represented by tock and step, which all processes synchronise on. When the possible actions are complete, processes will agree to "step". Once there is no progress that can be made within the system, a "tock" is produced to introduce possible external changes. Another global synchronous event calculate is introduced after each tock and step. It occurs after all external inputs or effects of the previous step have propagated themselves. The sequence of these three actions is shown in Fig. 2.

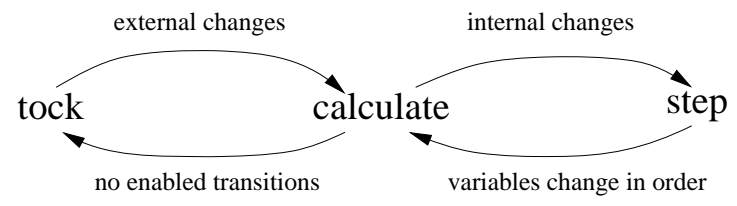

Fig. 2. Sequence of tock, step and calculate

After a super-step, changes can be made to the inputs, and timers are advanced by one time unit. Inputs and changes to timers only happen on supersteps. After this we get as many small steps as are required until no further progress is possible, i.e., there are no possible transitions without external changes made by the environment. Inputs and timers do not change during the execution of a small step. Within a small step, internal changes can be made to variables, which is done by communicating with the variable processes.

\subsection{Hierarchical Structuring}

Statecharts are structured, and one chart can sit inside a single state of another. The inner one is active only when the enclosing state is, and is turned off if the enclosing one is left.

At the highest level the model of a chart combines three synchronous processes: Sys represents the hierarchy of charts, VARSandTIMERS holds the values of variables and timers, and the combination of NOACT and TOX to enforce the timing model and outputs. At the highest level a chart is defined as following:

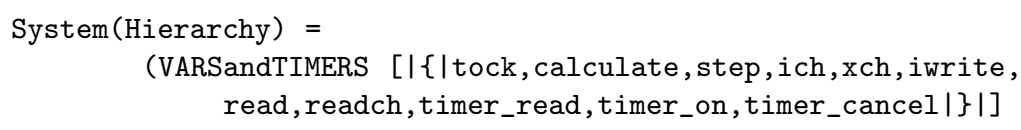




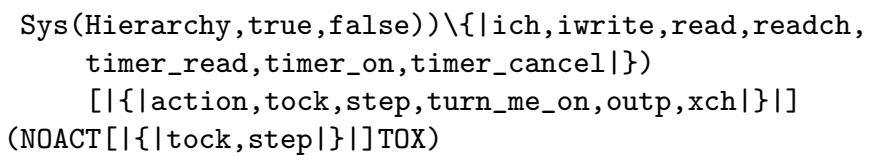

The parameter Hierarchy must be defined in the user script or as output from a higher-level tool. The second parameter of Sys is set to "true" meaning that the highest level machine in the hierarchy is initially on.

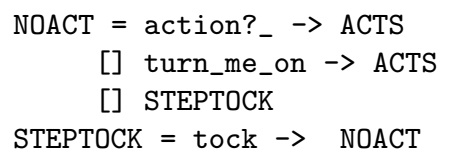

The step semantics is achieved through synchronization with the NOACT process which obliges a step to happen as opposed to a tock just when there has been an action since the last step or tock. The highest level chart has no enclosing state which can turn it on and off, so we forbid the actions "turn_me_on" and "turn_me_off".

ncSYSTEM (Hierarchy) $=$ System (Hierarchy)

[|\{|turn_me_on,turn_me_off $\mid\} \mid]$ STOP

Hierarchy The definition of the main type of chart structures is:

datatype Statechart $=$

SCTree.SGlabel.Set (ActLabel).Set ((Statelabel, Statechart))

This recursive definition means that a chart is root behaviour identified by one of these labels, a set of actions labels that are not to be promoted beyond this point, and a set of pairs, each a state label of the present chart with a chart which sits within it. We defined a function Sys ()$^{3}$ recursively that produces the CSP representation of statechart. A chart with no lower level charts is compiled using the function Machine ()$^{3}$, which creates a process from the description of a sequential chart. Each sequential machine has an event proceed which is used to enable subcharts to do things (implementing the priority rule). For the case with no sub-machines this action is not required, and therefore we prevent it by synchronisation with STOP. The actions are renamed to progress to discard information unnecessary outside this level and to square the outer alphabet of the process with what will be required outside.

Sys () is applied to all subcharts, which are then appropriately synchronised with each other and also with the top level machine which has itself been created with Machine(). We use many renamings and synchronisations to coordinate the behaviour of these parts.

\footnotetext{
${ }^{3}$ See full definitions of these functions in http://web.comlab.ox.ac.uk/oucl/work/bill.roscoe/publications/115.pdf .
} 


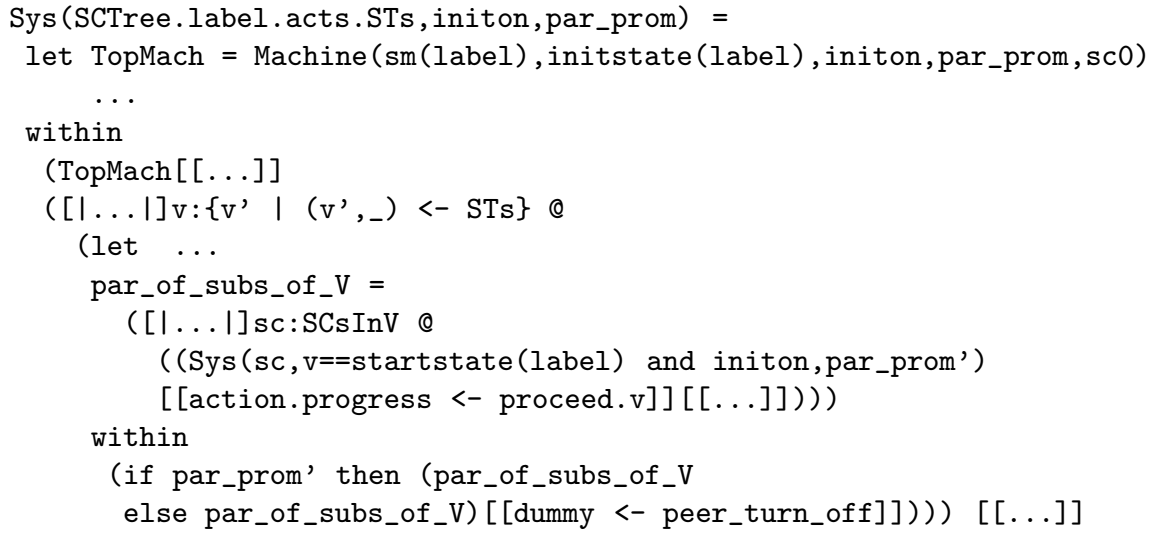

par_of_subs_of_V represents the construction for particular node's subcharts. Processes representing subcharts in different substates are synchronised, together with the TopMach ()$^{3}$. Actions progress by inner machines are controlled by events proceed, and all actions within this chart and not controlled outside is renamed to progress.

Implementing Priority and Promotion Standard CSP, as implemented in FDR has no priority. Any one of a set of the currently available actions can occur next, and this is never made impossible by another action being enabled. This is not the case in statecharts at several levels. It impacts upon the two-level model, and on the relative priorities of transitions at low-level, high-level and inter-level (promoted actions). Actions have names, but there is no synchronisation on them between charts. Our model handles this by giving sequential components an additional action progress which it perform precisely when there is nothing else it can do on a given step. It is then possible to synchronise high-level progress actions with low-level actions so that the latter only happen when no higherpriority action is possible [26].

Consider a high-level sequential machine whose states contain sub-statecharts that run while the given state is enabled. Our model treats these subcharts as separate parallel processes and so we need to link the behaviour of the highlevel state machine with the processes representing the subcharts. The model we adopt is to give each chart (other than, perhaps, the highest-level one) events "turn_me_on" and "turn_me_off" with the obvious effects. They are then (after suitable renaming) synchronised with the transitions of the high-level machine.

An inner chart may perform an action which is promoted to be an action of an outer one. Such an action must be named (not "progress") and any higher level chart that promotes it must use the same name. The actions of any state are divided into primary ones that the state instigates itself and secondary ones which are promoted by or through this state. To ensure charts running in parallel to one with an action that is promoted higher get turned off, the process Prom_Mon is introduced: 


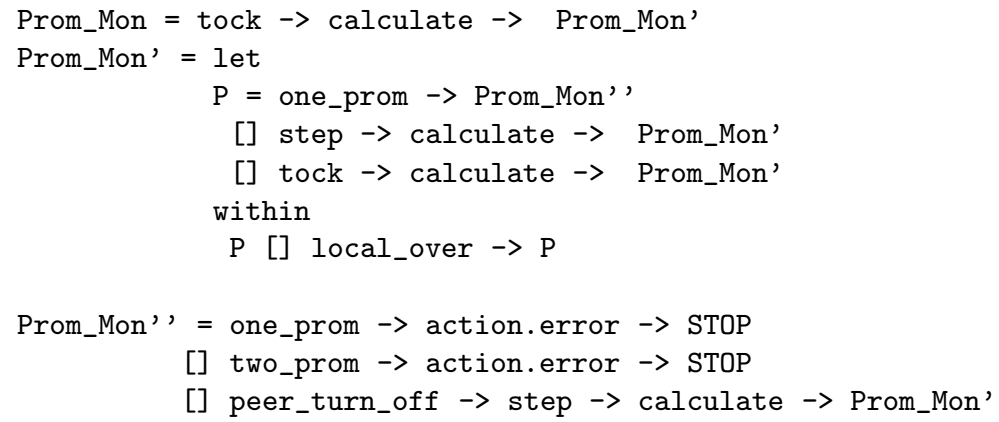

\subsection{Variables and Timers}

Variables are defined as processes running parallel with the system. The variable process implements many features of the semantics including the variable update model. The CSP process shown below codes a variable:

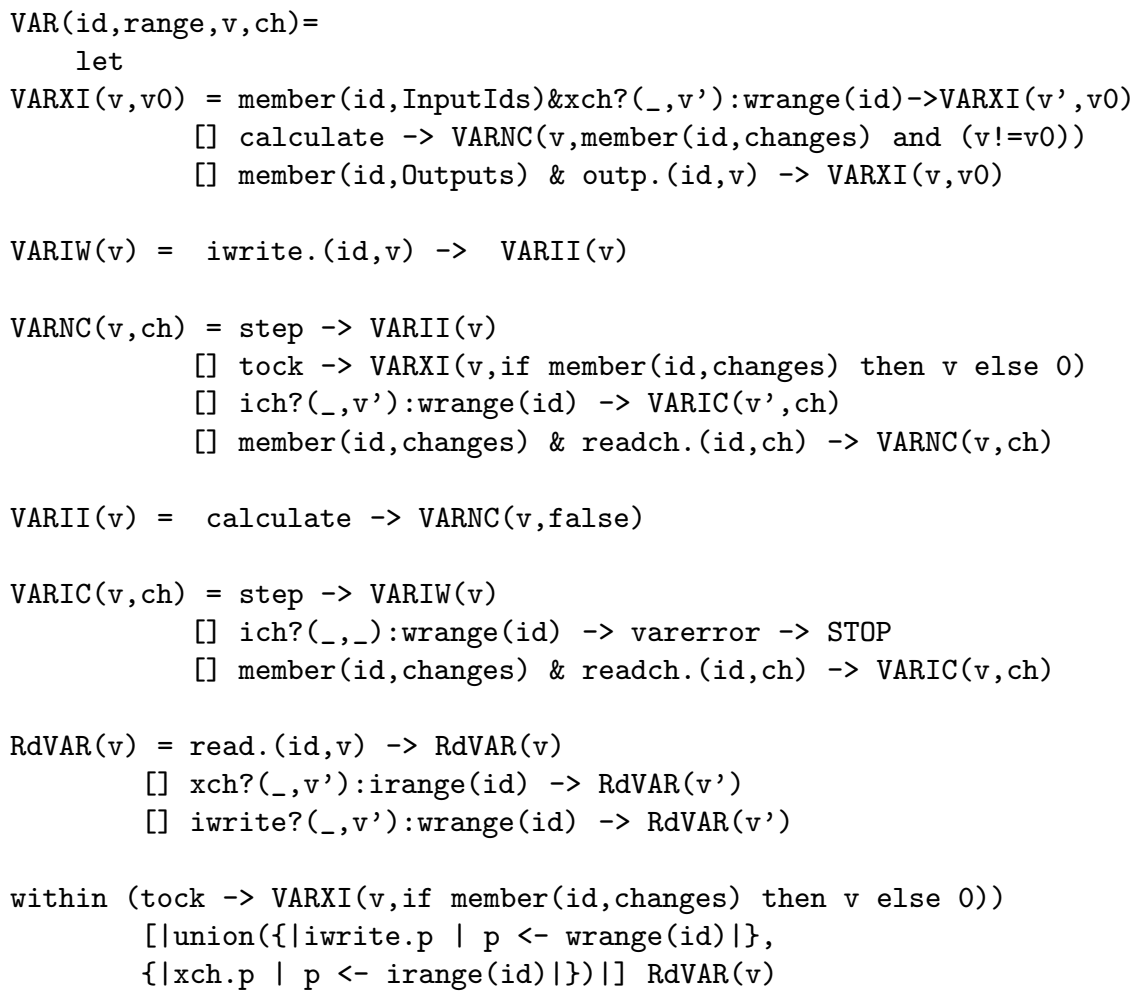

Comments:

- Channels: 
xch: the channel for external changes, changes the value of variable immediately, only possible on tock

ich: the channel for internal changes, informs processes of real internal changes of values after the calculation action

iwrite: the channel for internal writes, writes value variables, two of which on the same variable in one step are an error

outp: the channel for outputs, helps analyzing

read: reads values from variables

readch: checks for changes

- The purpose of the parallel composition with process RdVAR(v) is to allow a process to read the value of this variable from the previous step even after it has been changed via ich on this one. The new value is assigned to take effect after the variable has been changed via iwrite.

Note that our definition also has the properties that external inputs and outputs only occur immediately after tock and two internal changes on one step are an error. All variables are running in parallel, which produce a process for the entire set of variables:

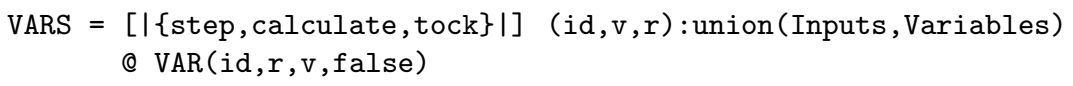

Timers are also defined as processes running parallel with the system. There are three kinds of timers: one is set up on the entry to a state; one is addressed by its own identifier name; another one is set up as a condition for transitions. A timer is initialized to zero and incremented by one each tock until the corresponding limit is reached. The CSP process shown below codes a straightforward timer:

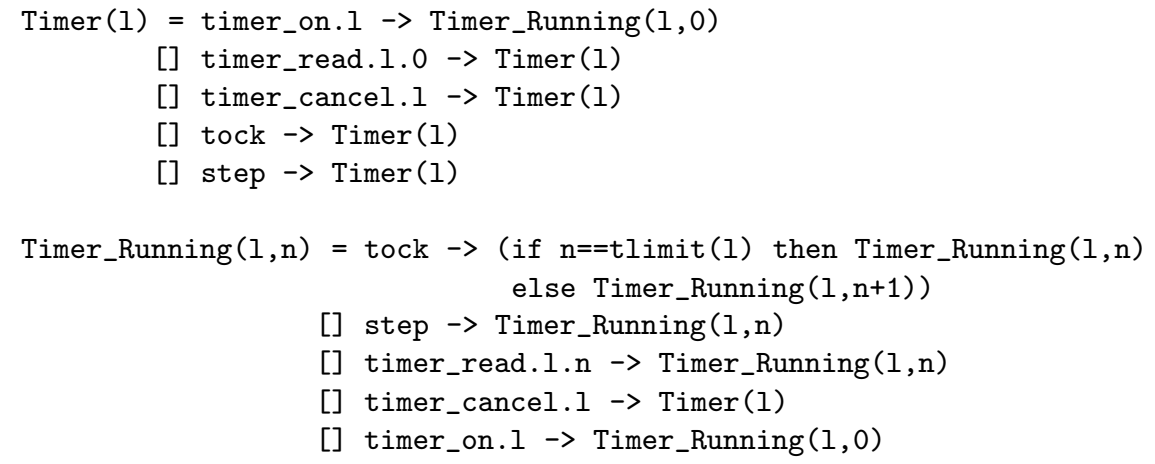

Comments on channels:

timer_on: turns the timer on, followed by initializing the timer to 0 timer_cancel: cancels the timer, remove the timer from the list of active timers

timer_read: reads the current value of the timer 


\subsection{Translating statecharts into CSP}

We analyse statecharts automatically by simulating them in CSP. In other words we have a CSP process which accepts the description of a statechart $S$ as a parameter and then behaves like $S$. We can prove things about $S$ by analyzing this simulation on FDR.

The way the simulation is written is therefore as a CSP program which closely resembles a translation from statechart syntax to behaviourally equivalent CSP. The combination of the simulation operation and FDR's own CSP compiler produces a compiler from statecharts to FDR internal state machine code. For simplicity we therefore refer to the code that creates the simulation as a compiler. The current definitions of the CSP language mean that it does not have good string processing. For that reason we supply the input statechart to it as a member of a specially designed CSP type plus various ancillary definitions of sets. In other words, by the time CSP sees the statechart, it has been parsed and symbolized, see Section 4 for more details of this CSP syntax.

\section{Specification Checking}

Many checks can be implemented after the entire system is built. Typically there are three types of checking: checks for general errors in the system, tests for reachability of states, and checks for consistency with application-specific requirements.

\subsection{Checks for Errors}

The simulation is written so that finitely detectable run-time errors are all flagged by an error event:

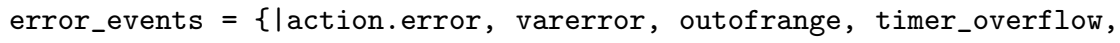

Event action. error indicates nondeterminism caused by ambiguous branching; event varerror indicates multiple writes on a small step; event outof range occurs when assigning a value which is out of range; event timer_overflow indicates the attempt to read a timer that has reached the bound limit; event type_error occurs when a boolean expression produces result not 0,1 .

The following standard check should be used for all charts:

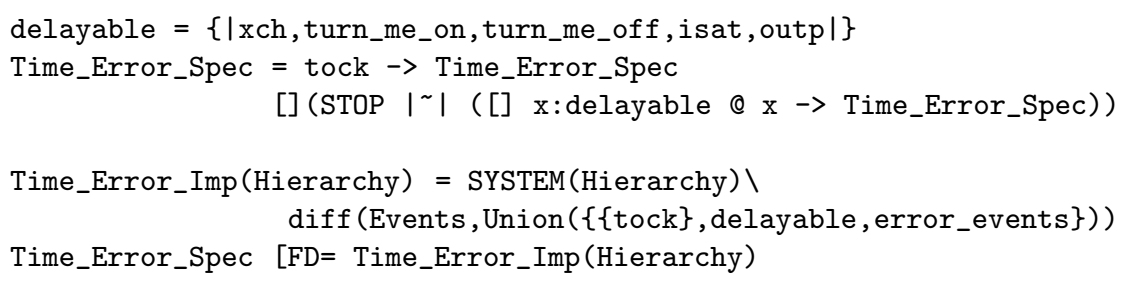


This refinement tests for all types of run-time errors, plus race conditions. ${ }^{4}$ It also ensures that there can never be an infinity of step events without an infinity of tocks. It is this timing aspect of the check that explains the name. This check uses the full power of failures/divergences refinement. However once it is satisfied all subsequent checks will normally be done. A large proportion of the errors we have discovered in industrial case studies have been via this check.

Race Condition Multiple writes to a variable on one small step lead to race condition and are considered as an error. As showed in the definition of variables in Section 2.5, an error message "varerror" would be returned by checking through FDR.

Nondeterminism Ambiguous enabled actions by any single process cause nondeterminism. Each sequential machine should have at most one action available at a time. Nondeterminism are considered as an error. If Nondeterminism exists in the system, an event "action.error" will be returned by checking it through FDR.

\section{$3.2 \quad$ Reachability}

To test whether a certain state in any chart can be reached at some point, the following refinement is used:

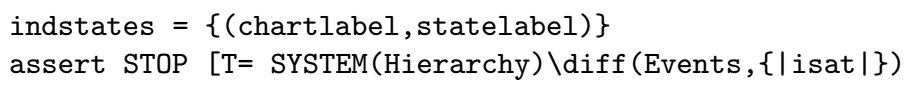

The set indstates ${ }^{5}$ is used to indicate the state we want to test for reachability. The refinement fails if and when the intended state is reached and a trace to describe how that state is reached can be provided by FDR. Note that this check does not behave ideally with attempts to prove things about a chart under non-deterministic parameters, since it will simply demonstrate that it is sometimes possible to reach the state, not that it is for all values of the parameter.

\subsection{Analysis of Properties}

Special requirements can be tested to ensure that they are satisfied by the system. Mostly one wants to check safety or security properties, which hold for the whole lifetime of the system. As already mentioned, we model the formal semantics of statecharts and requirements in the input script. Assume the chart representing the requirement is labeled by spec_label, the check can be made as follows:

\footnotetext{
${ }^{4}$ The check would fail if the simulation deadlocked. However deadlock is impossible because of the way the simulation is constructed. This feature was useful in designing the compiler.

${ }^{5}$ See full definitions of isat and indstates in http://web.comlab.ox.ac.uk/oucl/work/bill.roscoe/publications /115statechartcompiler.csp.
} 
SysAndSpec_sc $=$ SCTree.Box. \{\}$.\{(0$, Hierarchy $),(0$, lift (Spec_label $))\}$

assert STOP [T= SYSTEM(SysAndSpec_sc) \diff(Events, \{action.spec_error\})

The event action.spec_error indicates that the requirement is not satisfied.

\section{Case Study: Burglar Alarm System}

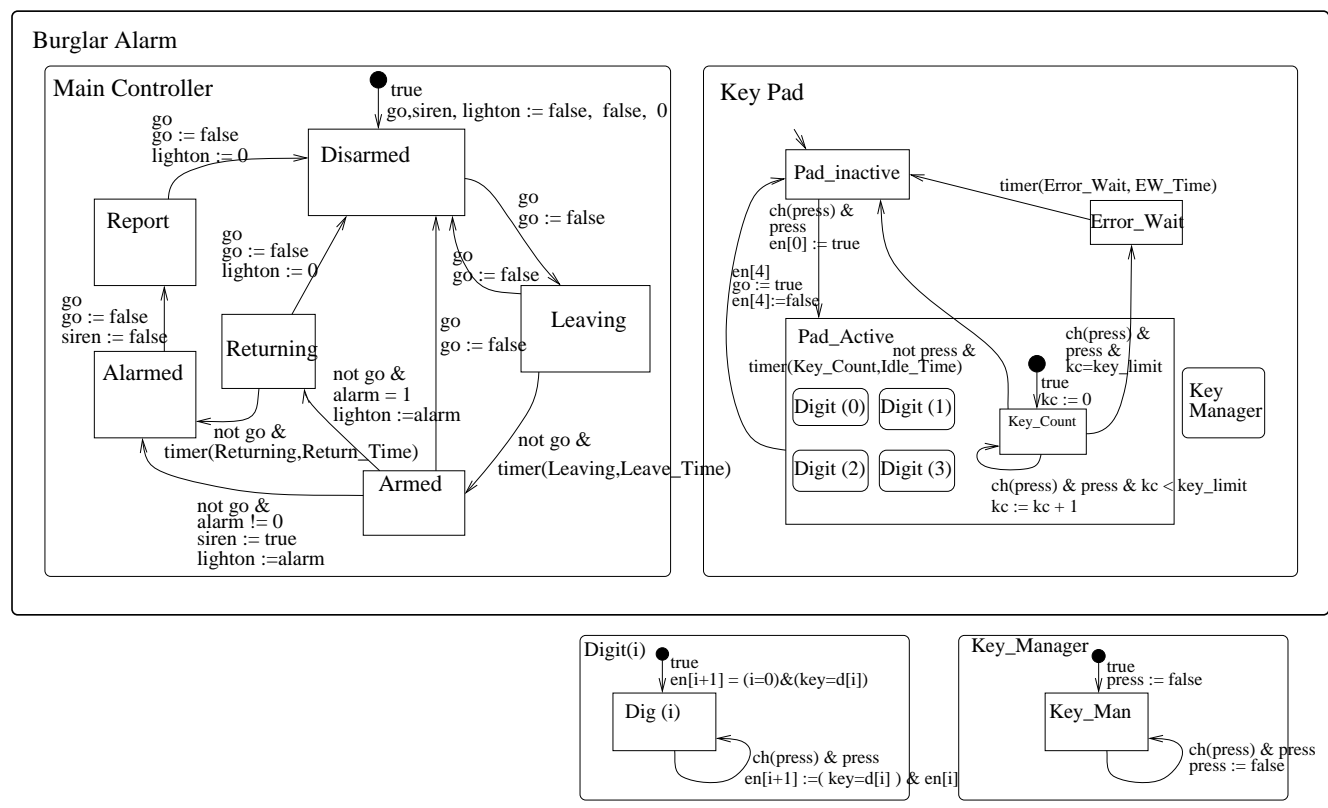

Fig. 3. Burglar Alarm System

A practical case study is the burglar alarm control system. The system provides a very typical example with the features like multiple parallel subcharts, inter-level transitions etc. (see Fig. 3)

The alarm is constructed of two nearly independent parallel processes: one is the number pad that determines (subject to things like timeout and too many attempts as limitations) when the last $D$ digits input are the code number for arming and disarming the alarm.

The Main Controller chart moves the alarm between its principal states: correctly typing in the code number (generating the go signal) will move it from disarmed to leaving (which times out to armed) and back to disarmed. If the alarm goes off we can type in the number once to turn off the siren and once to get rid of the signal light that tells us where the alarm came from. The state 
Returning gives some time to disarm the alarm if someone returns and is detected in the area of the controller box (Alarm zone 1). The state Leaving gives the user time to leave after arming the alarm.

The Key_Pad chart indicates that the pad becomes active when a key is pressed. It goes back to inactive if either the code is correctly entered or if too long has passed since the previous press. If more than $k c$ digits are used in an attempt to key the code, a wait is imposed. The five constituents of Pad_Active all run in parallel while that state is running, and similarly the main graph and Key_Manager. The chart Digit indicates that if the digit just pressed is $d[i]$ and the most recent digits pressed are $d[0] . . d[i-1]$ then enable the next process in the chain. The Key_Manager chart shows that press will become true each time the user enters a digit, and key takes the value pressed. The function of this process is to ensure that press is only true immediately after the users input so a user-change is always from false to true.

\subsection{Describing the System}

The following is part of a CSP file describing the statechart in Fig. 3. In practice the creation of these files is automated by a GUI that inputs details of the statechart from the user.

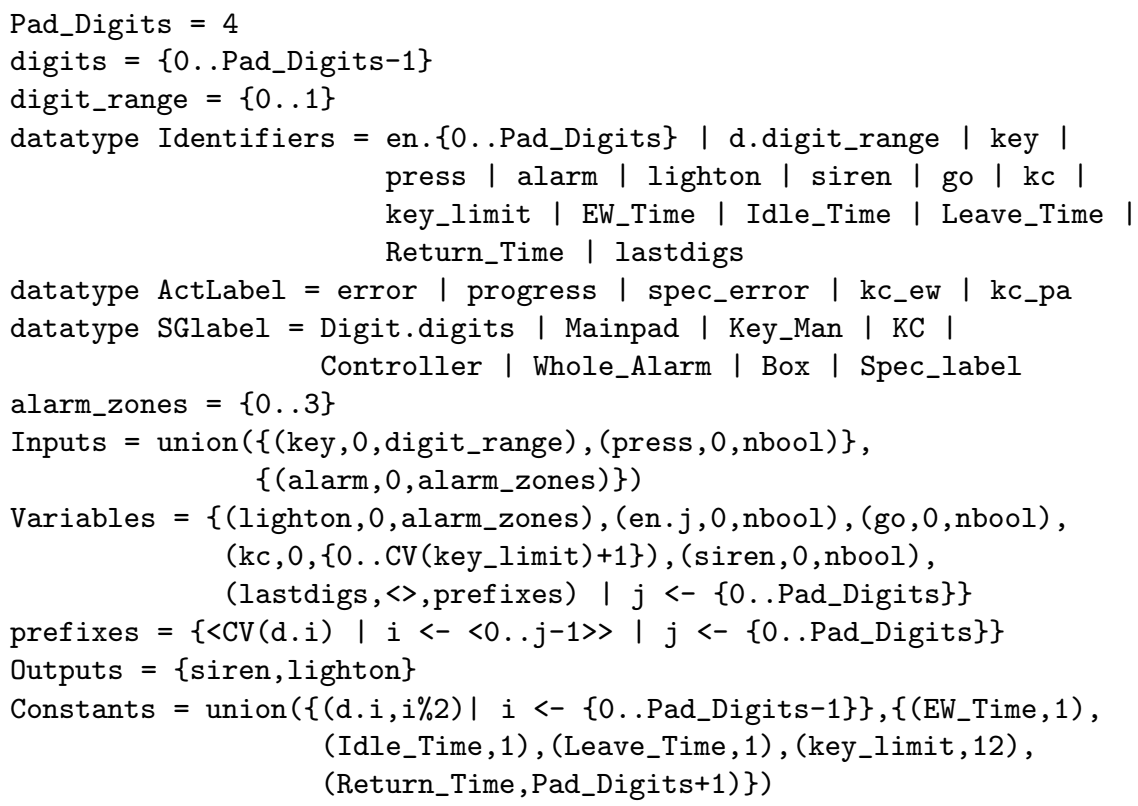

We formulate the corresponding process for chart Main Controller:

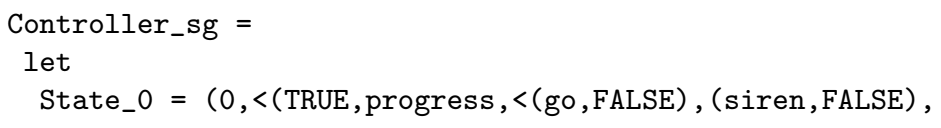




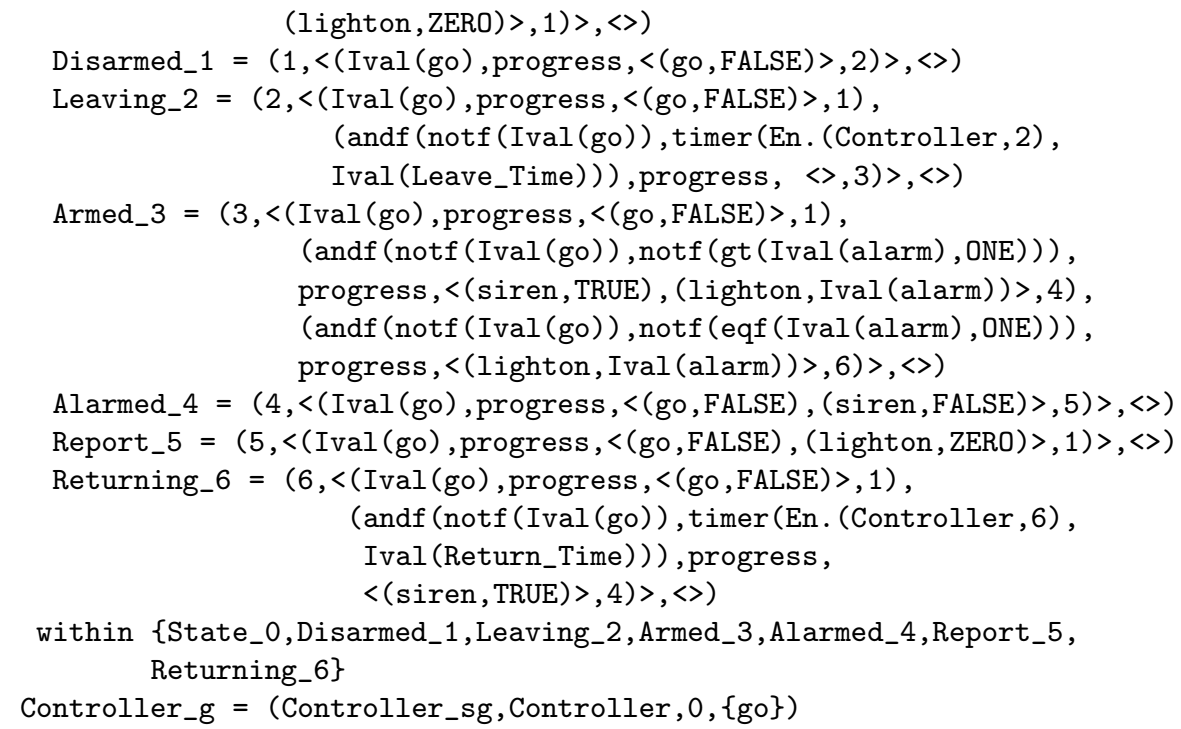

The processes for charts Key Pad, Digit, and Key Manager are defined similarly to this. We collect information together for whole system:

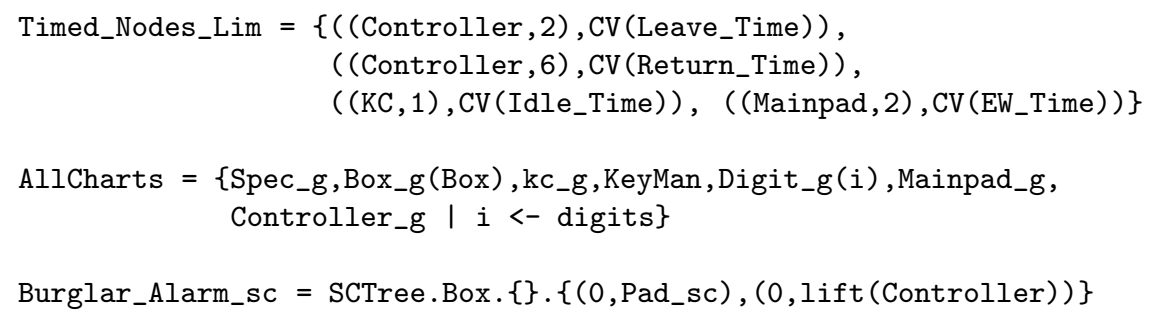

\subsection{Property Checking}

A property can be verified against the system by trace refinement of the corresponding CSP processes. There are two possible ways of verification depending on the representation of properties: representation as statecharts or implementation as CSP directly.

Statechart specification The first case means trivially using the existing translation. For showing an example of an analysis driven by the presented approach we use the statechart specification of a watch dog (Fig. 4). The requirement is: the siren never goes off until firstly the code number has been typed in and secondly an alarm signal has appeared. Now we translate this statechart into the input script for our compiler:

Spec_sg $=$

let 


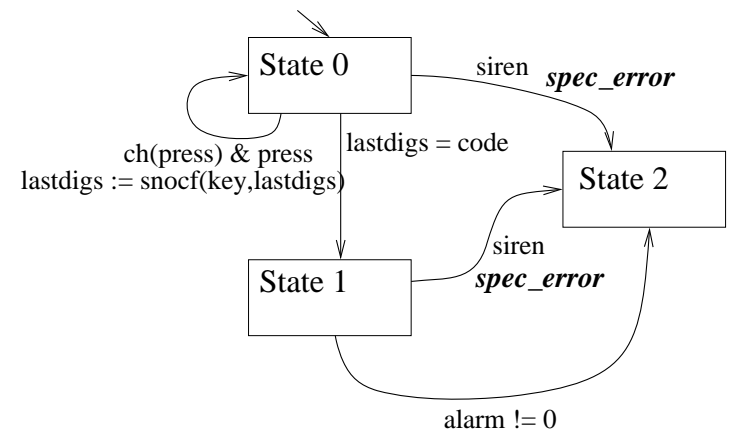

Fig. 4. A Watchdog Statechart

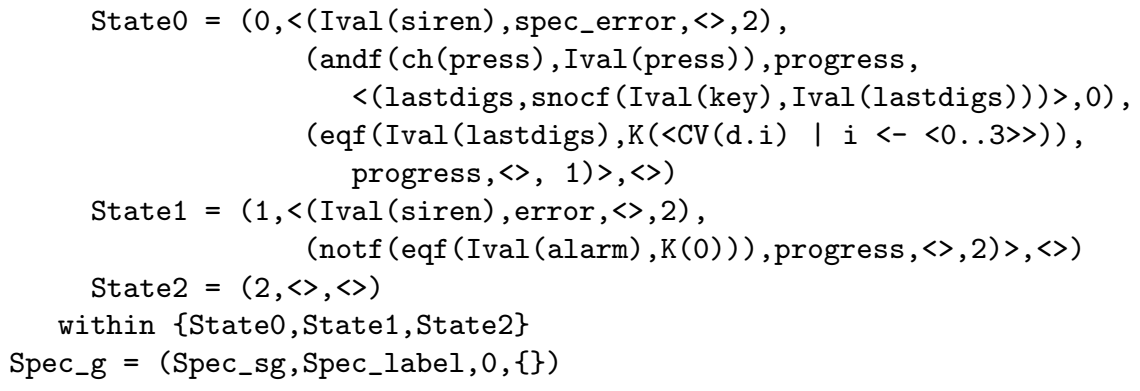

The specification shows that it moves from State0 to State1 when the digits have been correctly entered, and prior to that does the calculations to know when this has been done. It moves from State1 to State2 when alarm is not 0, and only then does it not raise an alarm when the siren does off. This is a statechart which is run in parallel with an implementation and can read its variables. It may not, however, write to any variable used by the implementation. Its function is to keep track (as it wishes) of what goes on and to raise a flag (spec_error) which can be caught by the Time and Error check if the implementation does something wrong. These conditions mean that it never interferes with the implementation.

SysAndSpec_sc =

SCTree.Box. \{\}$.\{(0$, Burglar_Alarm_sc $),(0$, lift (Spec_label) $)\}$

Now the task is to check the trace refinement relation between the property Spec_label and the system Burglar_Alarm. For that reason it is necessary to hide all the communication of SysAndSpec which is not spec_error.

assert STOP [T= SYSTEM(SysAndSpec_sc) \diff(Events, \{action.spec_error\})

The used model checker FDR executes the refinement check and returns the CSP model Burglar_Alarm meets the Property Spec_label:

Refine checked 199,245 states.

With 1025751 transitions.

True 
CSP-style specification We can specify our burglar alarm directly in terms of the events communicated by the CSP implementation. This particular model is not that good for this type of specification since the event of typing in a code number is rather diffuse (and almost certainly better handled using the watchdog style above). The following specification asserts that the siren cannot sound for at least $\mathrm{k}$ time units from the start:

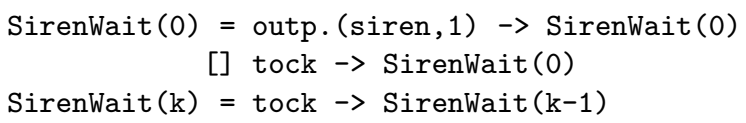

We can check this for various values via the trace check and the following are the (parameterised) limit.

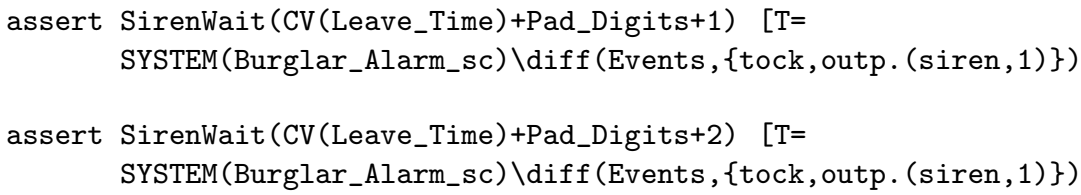

This is done by the used model checker FDR.

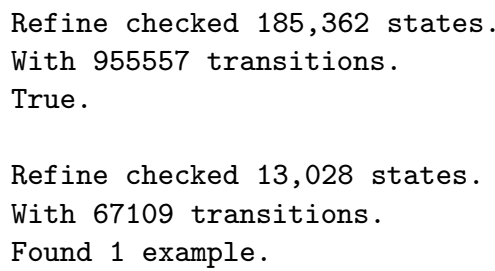

\section{Conclusion}

We have used the process algebra CSP and its model checker FDR to model and analyse Statemate Statecharts. Following this approach, the scope of some existing modeling techniques has been widened to address the problems that have arisen in various case studies. We have discovered many errors in practical industrial systems by this approach. For example, our compiler has been successfully used on an automotive system design project, the single lane architecture (QinetiQ), etc. Our compiler provides an efficient way to translate Statemate Statecharts to CSP and has proven to be sufficiently malleable to allow us to capture various properties of the semantics.

As a result of extensive research and studies in Statemate modelling, it is concluded that the idea of modelling statecharts in CSP has opened up, and will continue opening up, many opportunities for researches to model various graphic specifications which are widely used for complex systems. There is enormous scope for future development. The next target is MATLAB Stateflow (which is similar to statecharts). It is hoped that the demonstration of how to apply CSP theory to statecharts will inspire new approaches to standard graphic notations. 


\section{References}

1. R. Anderson, P. Beame, S. Burns, W. Chan, F. Modugno, D. Notkin, and J. Reese, Model checking large software specifications, IEEE Transactions on Software Engineering, Vol. 24, No. 7, pp. 498-520, 1998.

2. T. Bienmuller, J. Bohn, H. Brinkmann, U. Brockmeyer, W. Damm, H. Hungar, and P. Jansen, Verification of automotive control units, in E.-R. Olderog and B. Steffen (Eds.), Correct System Design, Springer Verlag, Berlin, 1999,number 1710 in LNCS, pp. 319-341.

3. T. Bienmuller, U. Brockmeyer, W. Damm, G. Dohmen, H.-J. Holberg, H. Hungar, B. Josko, R. Schlor, G. Wittich, H. Wittke, G. Clements, J. Rowlands, and E. Sefton, Formal verification of an avionics application using abstraction and symbolic model checking, in F. Redmill and T. Anderson (Eds.), Towards System Safety - Proceedings of the Seventh Safety-critical Systems Symposium, Huntingdon, UK, Safety-critical Systems Club, Springer Verlag, Berlin, 1999, pp. 150-173.

4. J.R. Burch, E.M. Clarke, and D.E. Long, Symbolic model checking with partitioned transition relations, In VLSI 91, Edinburgn, Scotland, 1990.

5. J.R. Burch, E.M. Clarke, K.L.McMillan, and D.L.Dill, Sequential circuit verification using symbolic model checking, In 27th ACM/IEEE Design Automation Conference, 1990.

6. J.R. Burch, E.M. Clarke, K.L.McMillan, D.L.Dill, and J. Hwang, Symbolic model checking: 10E20 states and beyond, In LICS, 1990.

7. T. Bienmuller, W. Damm and H. Wittke, The Statemeat Verification Environment - Making it real, In: Proc. CAV, LNCS 1855, pp. 561-561, Springer, 2000.

8. E.M. Clarke, O. Grumberg and D.E. Long, Model Checking and Abstraction, In proceedings of the Nineteenth Annual ACM Symposium on Principles of Programming Languages, 1992.

9. W. Damm and D. Harel, LSCs: breathing life into message sequence charts, in FMOODS'99 IFIP TC6/WG6.1 Third International Conference on Formal Methods for Open Object-Based Distributed Systems, Kluwer Academic Publishers, NY, 1999.

10. W. Damm, B. Josko, H. Hungar, and A. Pnueli, A compositiona real-time semantics of STATEMATE designs, in W.-P. de Roever (Ed.), Proceedings, International Symposium on Compositionality-The Significant Diference, Springer-Verlag, 1998, Lecture Notes in Computer Science.

11. W. Damm and J. Klose, Verification of a radio-based signalling system using the Statemate verification environment, Formal Methods in System Design 19:121-141, 2001.

12. H. Eshuis and R. Wieringa, A Formal Semantics for UML Activity Diagrams C Formalising Workflow Models, Technical Report, 2001.

13. Formal Systems (Europe) Ltd., Failures-Divergence Refinement, User Manual, obtainable from http://www.fsel.com/fdr2_manual.html

14. K. Fuhrmann and J. Hiemer, Formal Verification of STATEMATE-Statecharts, Citeseer.nj.nec.com/255163.html, 2001.

15. W.J. Fokkink and P. Hollingshead, Verification of interlockings: from control tables to ladder logic diagrams, in Proceedings of the 3rd Workshop on Formal Methods for Industrial Critical Systems-FMICS'98, Amsterdam. Stichting Mathematisch Centrum, 1998. 
16. K. Feyerabend and B. Josko, VIS: A visual formalism for real time requirement specifications, in Proceedings of the 4th International AMAST Workshop on RealTime Systems and Concurrent and Distributed Software, ARTS'97, Lecture Notes in Computer Science 1231, 1997, pp. 156-168.

17. T.V. Group, VIS: A system for verification and synthesis, in 8th international Conference on Computer Aided Verification, number 1102 in LNCS, 1996.

18. D. Harel and A. Naamad, The Statemate Semantics of Statecharts, Technical Report, i-Logix, 1995.

19. D. Harel, H. Lachover, A. Naamad, A. Pnueli, M. Politi, R. Sherman, and A.S. Trauring, Statemate: A Working Environment for the Development of Complex Reactive Systems, IEEE Transactions on Software Engineering, 16, 4, 1990.

20. J. Hudak, S.C. Dorda, D.P. Gluch, G. Lewis and C. Weinstock, Model-Based Verification: Abstraction Guidelines, Technical Note CMU/SEI-2002-TN-001, 2002.

21. D. Harel and M. Politi, Modeling Reactive Systems with Statecharts: The STATEMATE Approach, Part No.D-1100-43, i-Logix Inc., Three Riverside Drive, Andover, MA 01810, June 1996.

22. The MathWorks, Stateflow, User Manual, obtainable from http://www . mathworks.com/access/helpdesk/help/toolbox/stateflow/ug/

23. E. Mikk, Y. Lakhnech, C. Petersohn and M. Siegel, On Formal Semantics of Statecharts as Supported by STATEMATE, Technical Report, BCS-FACS Northern Formal Methods Workshop, 2, Ilkley, 1997.

24. L.E. Moser, Y. Ramakrishna, G. Kutty, P. Melliar-Smith, and L. Dillon, A graphical environment for design of concurrent real-time systems, ACM Transactions on Software Engineering and Methodology, Vol.6, No. 1, pp.31-79, 1997.

25. A.W. Roscoe The theory and practice of concurrency, Prentice-Hall International, 1998.

26. A.W. Roscoe, Compiling Statemate Statecharts into CSP and verifying them using FDR - abstract, Technical Report, 2003. 